\title{
Could insect phagocytic avoidance by entomogenous fungi have evolved via selection against soil amoeboid predators?
}

Correspondence
Michael J. Bidochka
bidochka@brocku.ca

Received 14 January 2010

Revised 18 March 2010

Accepted 19 March 2010

\author{
Michael J. Bidochka, ${ }^{1}$ David C. Clark, ${ }^{1}$ Mike W. Lewis ${ }^{2}$ \\ and Nemat O. Keyhani ${ }^{2}$ \\ ${ }^{1}$ Department of Biological Sciences, Brock University, St Catharines, ON L2S 3A1, Canada
${ }^{2}$ Department of Microbiology and Cell Science, University of Florida Gainesville, FL 32611-0700,
USA
}

The entomopathogenic fungi Metarhizium anisopliae and Beauveria bassiana are ubiquitously distributed in soils. As insect pathogens they adhere to the insect cuticle and penetrate through to the insect haemocoel using a variety of cuticle-hydrolysing enzymes. Once in the insect haemocoel they are able to survive and replicate within, and/or evade, phagocytic haemocyte cells circulating in the haemolymph. The mechanism by which these soil fungi acquire virulence factors for insect infection and insect immune avoidance is unknown. We hypothesize that insect phagocytic cell avoidance in $M$. anisopliae and $B$. bassiana is the consequence of a survival strategy against soil-inhabiting predatory amoebae. Microscopic examination, phagocytosis assays and amoeba mortality assays showed that these insect pathogenic fungi are phagocytosed by the soil amoeba Acanthamoeba castellanii and can survive and grow within the amoeba, resulting in amoeba death. Mammalian fungal and bacterial pathogens, such as Cryptococcus neoformans and Legionella pneumophila, respectively, show a remarkable overlap between survival against soil amoebae and survival against human macrophages. The insect immune system, particularly phagocytic haemocytes, is analogous to the mammalian macrophage. Our data suggest that the ability of the fungal insect pathogens $M$. anisopliae and $B$. bassiana to survive insect phagocytic haemocytes may be a consequence of adaptations that have evolved in order to avoid predation by soil amoebae.

\section{INTRODUCTION}

Insect pathogenic fungi, such as Metarhizium anisopliae and Beauveria bassiana, are considered to be facultative pathogens, since they survive within the soil, do not require an insect host for propagation, and yet maintain virulence (Roberts \& Hajek, 1992; Bidochka et al., 2000). Once isolated from soil, these fungi readily infect insects, and one of the most common methods of isolating them is to 'bait' the soil with an insect (Zimmermann, 1986; Bidochka \& Khachatourians, 1987). Virulence factors in these insect pathogenic fungi are not constitutively expressed and there are subsets of virulence-related genes upregulated during the various stages of insect infection (Wang et al., 2005). A critical unanswered question arising from the ecological niche and pathogenic nature of these fungi is how virulence is selected for and maintained in the environment. This issue is further complicated by the fact that most isolates of M. anisopliae var. anisopliae and B. bassiana demonstrate

Abbreviations: DIC, differential interference contrast; SEM, scanning electron microscopy; TEM, transmission electron microscopy. little or no insect host specificity. They are able to infect $>1000$ different insect and other arthropod species from various orders (Roberts \& Hajek, 1992; Bidochka et al., 2001, 2002).

Infection by M. anisopliae and B. bassiana begins when the fungal conidium contacts the insect cuticle, initiating the expression of genes involved in cuticle adhesion and degradation (Holder \& Keyhani, 2005; Cho et al., 2006a, b; Holder et al., 2007). The conidium germinates and the fungal hyphae transgress the insect cuticle, gaining access to the insect haemolymph. Here, another subset of virulence-related genes is upregulated, particularly those involved in differentiation and insect immune avoidance (Wang et al., 2005). Similar to the mammalian innate immune system, certain insect haemocytes are analogous to macrophages in that they encapsulate invading pathogens. However, M. anisopliae and B. bassiana have evolved mechanisms by which they avoid haemocyte encapsulation (Hou \& Chang, 1985; Gotz \& Boman, 1985; Bidochka \& Khachatourians, 1987; Pendland et al., 1993; Wang \& St. Leger, 2006). In an in vitro model system, these fungi can 
be phagocytosed by host cells, within which the fungal cells can survive and grow, eventually erupting from the host cell (Kurtti \& Keyhani, 2008). Human pathogenic fungi such as Cryptococcus neoformans, Blastomyces dermatitidis, Histoplasma capsulatum and Sporothrix schenckii appear to have evolved similar traits, being able to survive mammalian macrophage phagocytosis (Steenbergen et al., 2004).

There is evidence to suggest that the survival and growth of mammalian fungal pathogens within a macrophage evolved as a result of environmental pressures from soil-borne predators, particularly phagocytic amoebae (Steenbergen et al., 2001). The mammalian pathogenic fungus $C$. neoformans has been shown to survive phagocytosis, and to grow within the amoeba Acanthamoeba castellanii (Malliaris et al., 2004). Amoebae, human macrophages and insect haemocytes share common properties. They phagocytose their host and digest them with lysosomal enzymes and superoxidases (Davies et al., 1991; Bergin et al., 2005; Scully \& Bidochka, 2006). Here, we hypothesize that the mechanisms by which insect haemocyte avoidance evolved in insect pathogenic fungi are analogous, if not directly similar, to the evolution of avoidance of soil-borne phagocytic amoebae by mammalian pathogenic fungi. Using green fluorescent protein (GFP)-transformed B. bassiana and M. anisopliae isolates, the interaction between A. castellanii and the fungi was examined. In this study, we show that amoebae actively internalize the fungal conidia; however, the fungi are able to survive, germinate and grow within the amoebae, eventually erupting from the host cells.

\section{METHODS}

Strains and culture conditions. Stock cultures of $M$. anisopliae ARSEF 2575 and B. bassiana ARSEF 252 and ATCC 90517 were maintained at $-80{ }^{\circ} \mathrm{C}$ in $25 \%(\mathrm{v} / \mathrm{v})$ glycerol. In one set of experiments we used two additional fungal species, Alternaria alternata UAMH 10047 and Penicillium chrysogenum UAMH 6742. Cultures were grown on potato dextrose agar (PDA; Becton, Dickinson and Company) at $27{ }^{\circ} \mathrm{C}$ for 10 days to obtain conidia. Conidia were dislodged from the culture with $10 \mathrm{ml} 0.01 \%$ Triton-X/ PBS, and the suspension was poured through a glass funnel stuffed with glass wool to remove mycelial fragments from the conidial suspension. A stock culture of Saccharomyces cerevisiae was maintained at $-80{ }^{\circ} \mathrm{C}$. Cultures were grown for 4 days in peptone/yeast extract/glucose (PYG) broth in a shaker at 250 r.p.m. at $27{ }^{\circ} \mathrm{C}$.

The construction of GFP-expressing plasmids and transgenic fungal lines has been described previously (Fang et al., 2006).

A. castellanii strain 30234 (ATCC) was maintained in $100 \mathrm{ml}$ tissueculture flasks (Falcon; Becton Dickinson Labware) with $10 \mathrm{ml}$ PYG broth at room temperature on a shaker at 45 r.p.m. The amoeba cultures were maintained by diluting the amoeba medium by one-half with fresh medium every 5-7 days.

The extracted conidia, yeast and amoebae were washed by centrifuging the suspensions at $320 \mathrm{~g}$ for $10 \mathrm{~min}$. The supernatant was decanted and cultures were suspended in PBS. The process was repeated twice with the final suspension of $M$. anisopliae, B. bassiana, Alternaria alternata, P. chrysogenum or S. cerevisiae suspended in
$0.01 \%$ Triton-X/PBS, and A. castellanii suspended in PBS. The suspensions were diluted and then counted using a haemocytometer.

Amoeba killing assay. The fungal killing assay was based on a previously described protocol (Malliaris et al., 2004), with modifications. A. castellanii was diluted to $1 \times 10^{4}$ cells $\mathrm{ml}^{-1}$ and counted using a haemocytometer. Amoebae were added to wells of a 96-well plate at $200 \mu \mathrm{l}$ per well and allowed to adhere to the well for $1 \mathrm{~h}$ at $27{ }^{\circ} \mathrm{C}$ before $200 \mu \mathrm{l}$ of $1 \times 10^{4}$ cells $\mathrm{ml}^{-1}$ of M. anisopliae, B. bassiana or S. cerevisiae was added to produce a $1: 1$ effector-to-target ratio. The 96-well plates were then incubated at $27{ }^{\circ} \mathrm{C}$ for $0,8,16$ and $24 \mathrm{~h}$. $S$. cerevisiae was used as the positive control, since it has been found to be phagocytosed and rapidly killed by A. castellanii (Steenbergen et al., 2001).

The viability of $A$. castellanii grown under the experimental conditions was determined using a Trypan blue exclusion assay at each time interval. Fifty microlitres of a 1:20 dilution of Trypan blue stain was added to wells and the percentage of dead amoebae was determined by counting the number of amoeba cells unable to exclude the dye per 50 amoebae counted. The control for the Trypan blue counts consisted of wells containing A. castellanii with $0.01 \%$ Triton/PBS instead of fungi or yeast.

In the remaining wells the contents were pulled through a 27-gauge syringe needle five to seven times to completely lyse the amoebae and release the contents. The contents of each well were then plated onto PDA agar for M. anisopliae and B. bassiana, and YPD agar for $S$. cerevisiae, so that approximately $10^{2}$ cells were placed on each plate. The plates were then incubated at $27{ }^{\circ} \mathrm{C}$ for fungal c.f.u. determination. The control for c.f.u. determination consisted of wells containing M. anisopliae, B. bassiana and S. cerevisiae with PBS. In this experiment, conidia of $P$. chrysogenum and Alternaria alternata were also used as controls to determine whether survival of amoebic phagocytosis is a general property of soil-borne fungi.

A. castellanii phagocytosis of fungi. A. castellanii was diluted to $1 \times 10^{6}$ cells $\mathrm{ml}^{-1}$ with PBS. Ten microlitres of the A. castellanii suspension was placed on a microscope slide. M. anisopliae and $B$. bassiana were diluted to $1 \times 10^{6}$ cells $\mathrm{ml}^{-1}$ using a haemocytometer and $0.01 \%$ Triton-X/PBS. Ten microlitres of the fungal solution was placed on the slide with $A$. castellanii and the cell suspensions on the slide were mixed using a micropipette. The slide was placed within a Petri dish, which was wrapped with Parafilm (to prevent desiccation) and placed in an incubator at $27^{\circ} \mathrm{C}$. At 2, 4, 6, 8, 10 and $12 \mathrm{~h}$, the microscope slide was taken out of the Petri dish and the percentage of amoebae which had engulfed fungi was recorded. Samples for microscopic examination were prepared by seeding the amoebae in four-well LabTek chambered coverglass systems (Nunc). In some instances, extracellular conidia were separated from amoebainternalized conidia by Centricoll density-gradient centrifugation. Cultures of co-incubated fungi and amoebae were harvested and immediately applied on top of a step gradient of 25 and $50 \%$ Centricoll. Centrifugation was performed in sterile $2 \mathrm{ml}$ tubes, at $10000 \mathrm{~g}$ for $10 \mathrm{~min}$ at $4{ }^{\circ} \mathrm{C}$ using a table-top centrifuge. Amoebae (including those harbouring fungal conidia) were separated from external fungal cells and collected within the top portion ( $25 \%$ step) of the gradient (fungal conidia formed a pellet at the bottom).

Galleria mellonella (Wax Moth) larvae bioassays. A. castellanii

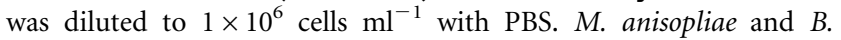
bassiana were diluted to $1 \times 10^{6}$ cells $\mathrm{ml}^{-1}$ with $0.01 \%$ Triton-X/PBS. Five-hundred microlitres of A. castellanii or PBS was placed into an Eppendorf tube labelled 24, 48 or $72 \mathrm{~h}$. Five-hundred microlitres of $M$. anisopliae or B. bassiana was placed into the Eppendorf tube containing the amoebae or PBS. The Eppendorf tubes were stored at $27{ }^{\circ} \mathrm{C}$ for $24 \mathrm{~h}$. After $24 \mathrm{~h}$, the tubes were removed from the incubator and the tubes containing $A$. castellanii were lysed using a 
vortex at full speed for $30 \mathrm{~s}$. The Eppendorf tubes, at $24 \mathrm{~h}$, were plated onto PDA agar so that approximately $10^{2}$ cells were plated. After 48 and $72 \mathrm{~h}$, the tubes were centrifuged for $10 \mathrm{~min}$ at $320 \mathrm{~g}$. The pellet was resuspended with $A$. castellanii or PBS to a volume of $1 \mathrm{ml}$ and stored for another $24 \mathrm{~h}$ at $27{ }^{\circ} \mathrm{C}$. The process was repeated twice more until the $72 \mathrm{~h}$ sample was plated and stored at $27{ }^{\circ} \mathrm{C}$. The fungal c.f.u. after 24, 48 and $72 \mathrm{~h}$ was recorded after 5 days of incubation at $27{ }^{\circ} \mathrm{C}$. The c.f.u. counts were used to dilute solutions of M. anisopliae and B. bassiana to $1 \times 10^{4}$ cells $\mathrm{ml}^{-1}$ to inject into G. mellonella. G. mellonella was injected with $3 \mu \mathrm{l}$ PBS, original conidial suspension, conidia passaged through PBS for three $24 \mathrm{~h}$ cycles, or conidia passaged through A. castellanii for three $24 \mathrm{~h}$ cycles. The G. mellonella were individually placed in snap-lid containers and kept at $27^{\circ} \mathrm{C}$, and mortality was checked daily.

Microscopy. Fixed-cell, live and time-lapse imaging was obtained using a Zeiss Axiovert Pascal LSM5 inverted microscope equipped with a laser scanning unit (for GFP, excitation $488 \mathrm{~nm}$, emission $505 \mathrm{~nm}$ ). Scanning electron microscopy (SEM) and transmission electron microscopy (TEM) were performed using Hitachi S400 and Zeiss EM10-CA electron microscopes, respectively.

\section{RESULTS}

\section{Uptake of M. anisopliae by A. castellanii}

Fluorescence microscopy was used to examine the interaction between GFP-transformed M. anisopliae and A. castellanii (Fig. 1). Superposition of differential interference contrast (DIC) and fluorescence microscopic images of the same sample showed co-localization of fungal cells within the amoebae, with increasing numbers of fungal conidia co-localizing within the amoebae over time. 3D (Z-stack) imaging was consistent with internalization of the conidia within the amoebae. Time-lapse imaging showed predation

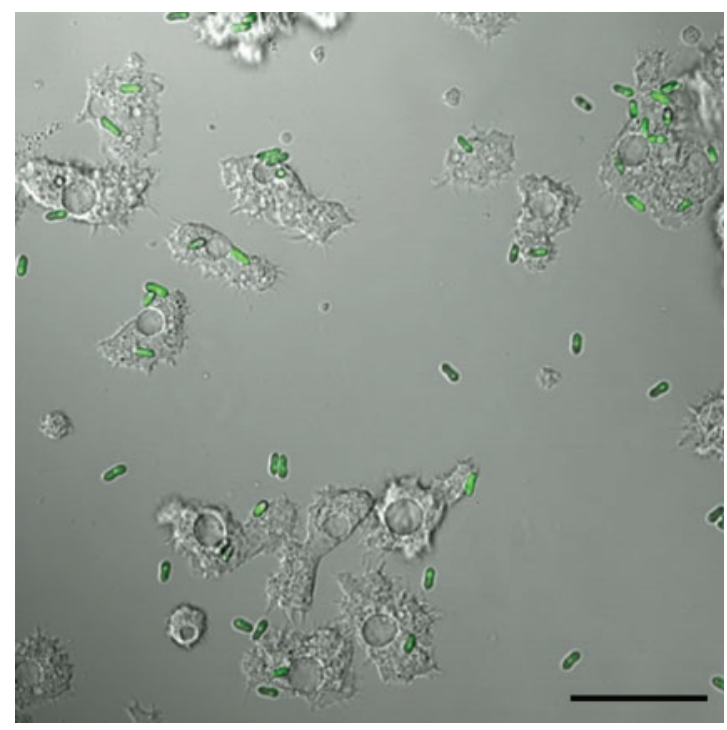

Fig. 1. $M$. anisopliae GFP-labelled conidia incubated with A. castellanii at $1-3 \mathrm{~h}$. Bar, $25 \mu \mathrm{m}$. of the fungal cells by the motile amoebae, and in several instances multiple conidia could be seen in different planes of focus within the amoebae. Experiments using infection ratios ranging from 0.1 to 10 fungal conidia per amoeba gave essentially the same results, with greater numbers of internalized conidia per amoeba visible as the fungal: host cell ratio or m.o.i. increased. Interestingly, qualitative observations of the amoebae indicated that amoebae that were actively preying upon the fungal cells tended to take up multiple conidia.

To show the internal nature of the fungal cells, SEM and TEM were employed. SEM images of the interactions between fungal cells and the amoebae indicated initial adhesion and subsequent engulfment of the fungal conidia by the amoebae, with pseudopodial interactions evident (Fig. 2). Internalization of fungal conidia by the amoebae was confirmed by TEM, in which samples were examined $3 \mathrm{~h}$ post-inoculation of fungal conidia with the amoebae (Fig. 3). In some instances, internalized conidia appeared to be enclosed within an endosomal compartment, although this was not always apparent. Qualitative analysis indicated that uptake increased over time, with amoebae often taking up multiple conidia. Some fungal conidia were clearly in large vacuoles, although a membrane surrounding all internalized fungal conidia was not always evident. To examine whether germination of internalized fungi could be observed by TEM, amoebae were allowed to prey upon the fungal conidia for $3 \mathrm{~h}$, after which external fungal conidia were removed by density-gradient centrifugation, and the amoebae were replaced in media for $18-20 \mathrm{~h}$, before samples were prepared for TEM analysis as described in Methods. Using such a protocol, fungal germination within the amoebae was observed (Fig. 3e, f).

\section{Quantification of uptake and amoeba killing by $B$. bassiana and $M$. anisopliae}

To quantify and measure the rate of uptake of the fungal conidia by the amoebae, GFP-expressing B. bassiana and $M$. anisopliae were employed to follow the time-course of amoebic phagocytosis. Fungal conidia were phagocytosed by amoebae within $2 \mathrm{~h}$ of co-incubation and this increased up to $8 \mathrm{~h}$ (Fig. 4 ). After $8 \mathrm{~h}$, the percentage of amoebae containing fungal conidia decreased. Between 8 and $16 \mathrm{~h}$ we observed a decrease in amoebae containing fungal conidia. This concurs with the onset of germination by fungi and an increase in amoeba mortality (see below).

Trypan blue exclusion assays were performed to determine the percentage of amoebae killed by the fungi. These data showed that amoebae were killed when incubated with $B$. bassiana and M. anisopliae but not S. cerevisiae (Fig. 5). When the assay was initiated, $>97 \%$ of the amoebae were alive and excluded Trypan blue. After $24 \mathrm{~h}$ incubation in PBS, $\sim 90 \%$ of the amoebae remained viable. There was no statistical difference in amoeba mortality between the control (Ac) and amoebae incubated with $S$. cerevisiae ( $t$ test, $P>0.05$ ). After $24 \mathrm{~h}, B$. bassiana and M. anisopliae 


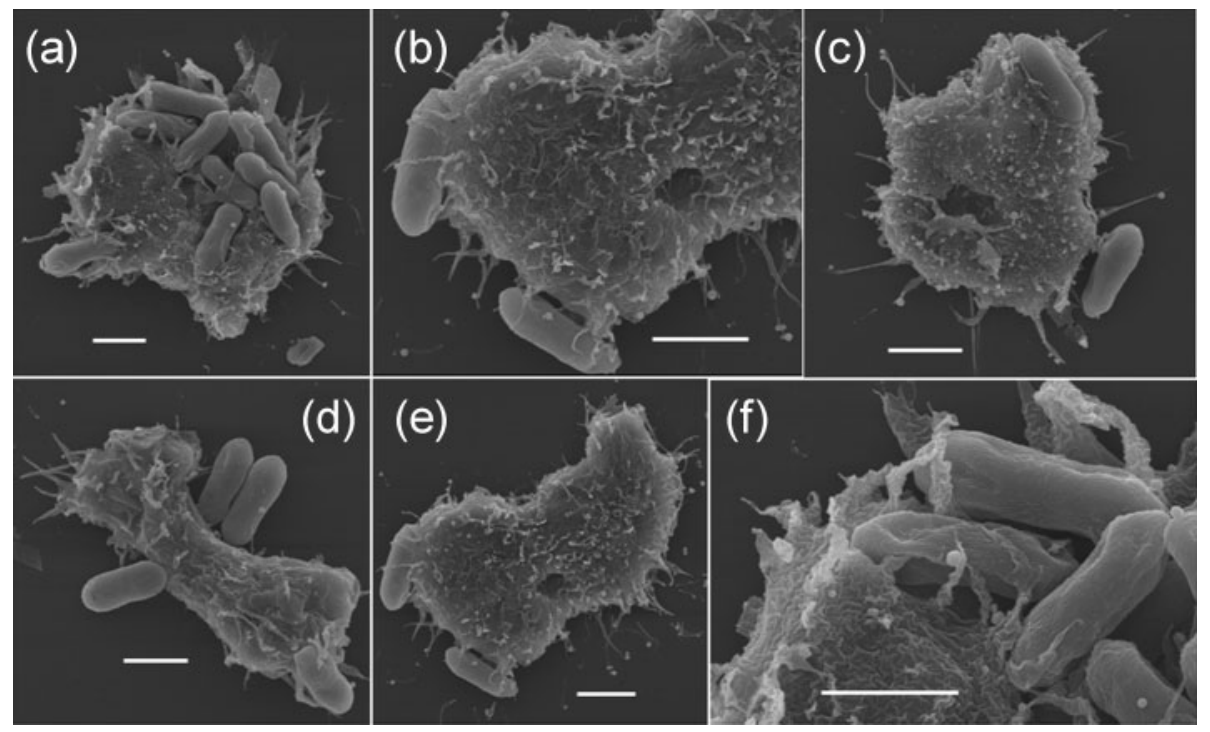

Fig. 2. SEM images of internalization of $M$. anisopliae by $A$. castellanii at $3 \mathrm{~h}$ incubation. Bars, $3 \mu \mathrm{m}$.

killed $20-22 \%$ of the amoebae, significantly greater than the control mortality ( $t$ test, $P<0.05$ ) or incubation with $S$. cerevisiae ( $t$ test, $P<0.05$ ). Cell-free extracts of $M$. anisopliae and B. bassiana incubated for $48 \mathrm{~h}$ in PBS then transferred to an A. castellanii culture in PBS did not result in increased amoeba death over the control.
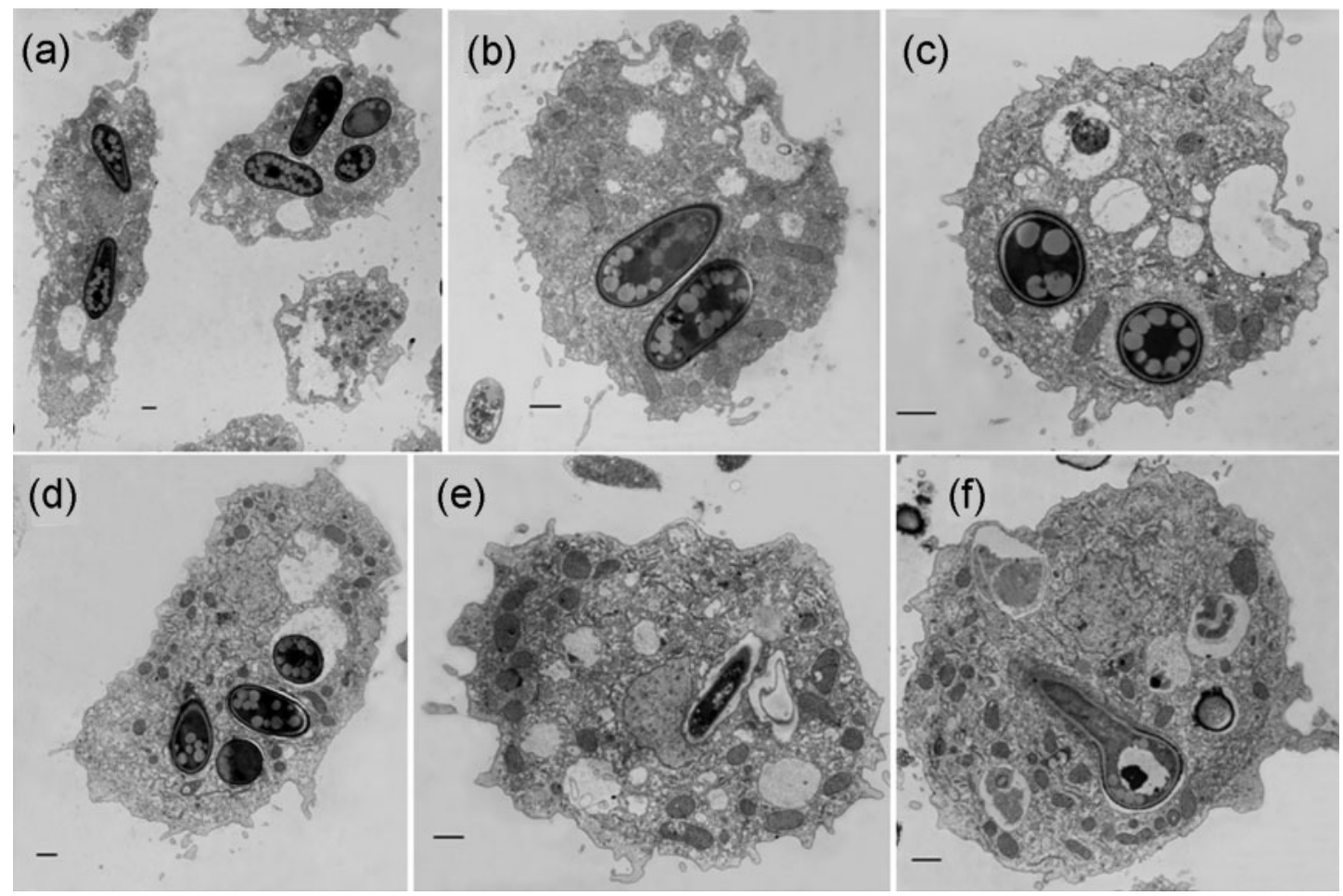

Fig. 3. TEM images of internalization of $M$. anisopliae by $A$. castellanii. (a-d) Samples fixed after $3 \mathrm{~h}$ co-incubation; (e, $\mathrm{f}$ ) coincubation for $3 \mathrm{~h}$, followed by harvesting and removal of external conidia, culture replaced at $28{ }^{\circ} \mathrm{C}$, and fixed after $18 \mathrm{~h}$ incubation. Bars, $6 \mu \mathrm{m}$. 


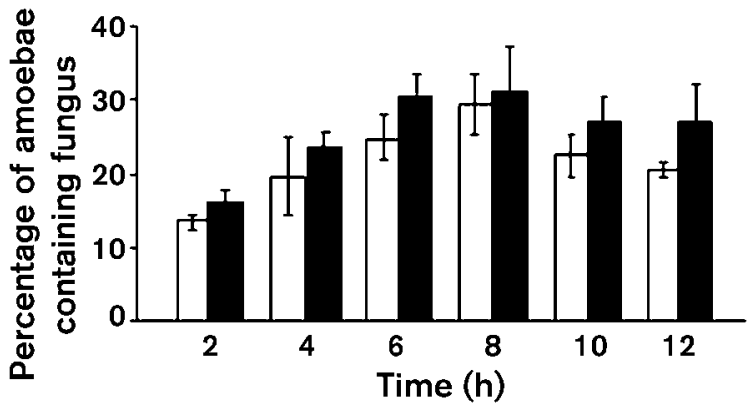

Fig. 4. Time-course of the proportion of amoebae containing $B$. bassiana (white bars) and M. anisopliae (black bars). For each time point, 200 amoebae were counted, with $n=5$. Error bars, SD. Data are representative of three separate experiments.

\section{Fungal growth in amoebae}

When B. bassiana, M. anisopliae, S. cerevisiae or the amoeba A. castellanii was incubated in PBS little growth or replication was noted. Both $B$. bassiana and $M$. anisopliae are dimorphic fungi able to grow as mycelia or, in the presence of nutrient-rich medium, they can evolve singlecell propagules termed blastospores. TEM images of fungal conidia internalized by the amoebae and allowed to incubate overnight revealed fungal germination within the host cell (Fig. 3e, f). Experiments performed using GFP-transformed M. anisopliae allowed for dynamic visualization of the fungal growth within the amoebae (Fig. 6). Hyphal emergence from amoebae containing the fungi was apparent, and in many instances several germlings could be seen growing within a single amoeba (Fig. 6).

To assess the extent of fungal growth in the presence of the amoebae, the number of fungal c.f.u. was determined over time during co-incubation experiments (Fig. 7). These data showed that when $M$. anisopliae or B. bassiana was incubated in the presence of $A$. castellanii, the total c.f.u. remained stable ( $t$ test, $P>0.05$ ). In contrast, a significant decrease in total $S$. cerevisiae c.f.u. was noted under similar conditions ( $t$ test, $P<0.05$ ). Here, we also showed a significant decrease in total $P$. chrysogenum and Alternaria alternata c.f.u. ( $t$ test, $P<0.05$ ). Microscopic examination showed that amoebae phagocytosed these fungi.
It should be noted that a germinated conidium undergoing hyphal growth would record as 1 c.f.u.; therefore, to obtain a better representation of the potential growth of the fungi in the presence of the amoebae, samples were vigorously vortexed to shear the fungal hyphae as well as the amoebae, and the resulting suspension was incubated with a fresh amoeba culture (Fig. 8). Under such conditions, increased growth, as seen by increased c.f.u., for M. anisopliae and $B$. bassiana was observed after three cycles of shearing and incubation ( $t$ test, $P<0.05$ ). In contrast, a decrease in $S$. cerevisiae c.f.u. was noted under identical conditions ( $t$ test, $P<0.05$ ), suggesting that $S$. cerevisiae is killed by the phagocytic amoebae, whereas $M$. anisopliae and B. bassiana are able to survive and grow.

\section{$B$. bassiana and $M$. anisopliae virulence is not enhanced by passage through $A$. castellanii}

We surmised that if $B$. bassiana and $M$. anisopliae survive insect haemocytic phagocytosis as a consequence of avoidance of amoebae in the soil, then virulence could also be affected by passage of fungal cells through amoeba cultures. To examine this possibility, fungal cultures were passaged three times through the amoebae as well as through PBS as a control. Insect bioassays using the larvae of the Wax Moth, G. mellonella, were subsequently performed by injection of $3 \mu$ of fungal material $\left(\sim 10^{7}\right.$ c.f.u. $\mathrm{ml}^{-1}$ ). From these experiments, median lethal time $\left(\mathrm{LT}_{50}\right)$ values for $B$. bassiana non-passaged, passaged through PBS and passaged through amoebae were determined to be 3.6, 3.3 and 3.2 days, and the $\mathrm{LT}_{50}$ for M. anisopliae non-passaged, passaged through PBS and passaged through amoebae was 2.1, 2.5 and 2.2 days. In each case, no significant differences in virulence were noted under the conditions tested.

\section{DISCUSSION}

Our data have led to the hypothesis that the selective pressure of soil amoeboid predation on M. anisopliae and B. bassiana may be critical in maintaining virulence factors that allow these fungi to survive insect phagocytic haemocytes. Using GFP-transformed strains of $M$. anisopliae and B. bassiana to investigate internalization, survival

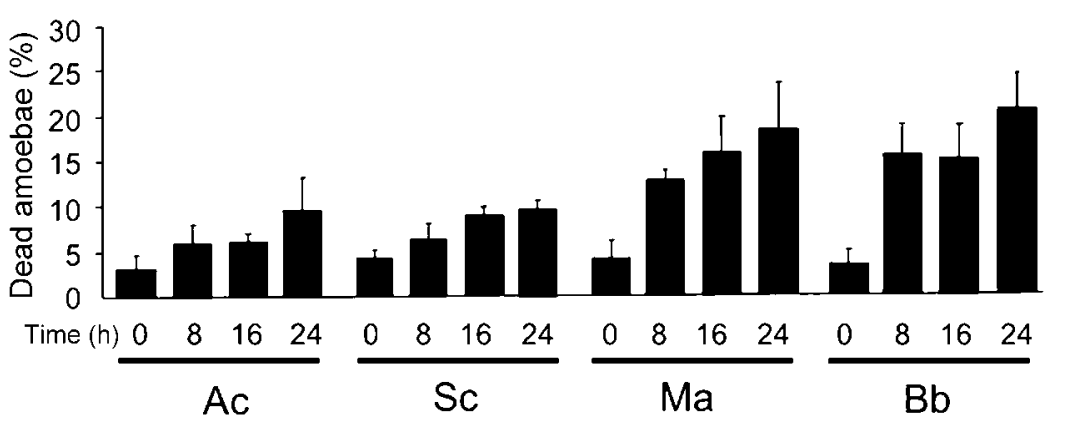

Fig. 5. Time-course of $A$. castellanii mortality, using a Trypan blue exclusion assay, during incubation with $S$. cerevisiae (Sc), M. anisopliae $(\mathrm{Ma})$ and $B$. bassiana $(\mathrm{Bb})$. Ac, control mortality. For each time point, 200 amoebae were counted, with $n=5$. Error bars, SD. Data are representative of three separate experiments. 


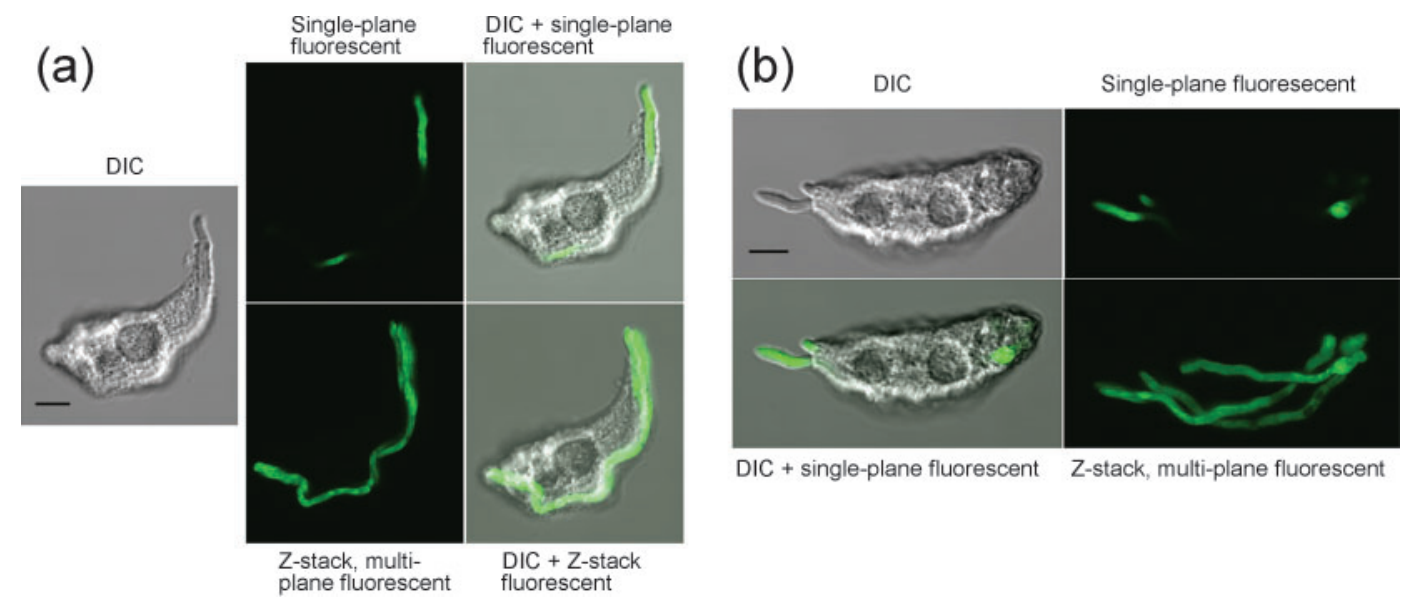

Fig. 6. DIC and fluorescence microscopy images. A low inoculum of $M$. anisopliae-GFP of $1: 50$ (M. anisopliae : amoeba) was employed, and the sample was incubated for $18 \mathrm{~h}$ at $28{ }^{\circ} \mathrm{C}$. Single- and multi-plane Z-stack images are shown. (a) and (b) show two separate examples. Bars, $10 \mu \mathrm{m}$.

and growth within the amoeba $A$. castellanii, our results show that M. anisopliae and B. bassiana are phagocytosed by amoebae but that these fungi are able to survive and grow within the amoebae, ultimately resulting in the death of the amoebae. After phagocytosis, some of the fungi were localized in membrane-bound vacuoles, and our data are consistent with the ability of $M$. anisopliae to survive phagocytosis by tick cell lines (Kurtti \& Keyhani, 2008). In contrast to the entomopathogenic fungi examined, the yeast $S$. cerevisiae was killed by amoebae, and earlier reports examining filamentous soil fungi including Aspergillus niger, Fusarium spp., Rhizoctonia solani, Sordaria fimicola and Stachybotrys atra have demonstrated that these organisms are unable to avoid amoebic phagocytosis (Old \& Darbyshire, 1978). We also showed that two common soil fungi, $P$. chrysogenum and Alternaria alternata, were also unable to avoid amoebic phagocytosis. Indeed, the phenomena of survival of amoeba predation may be restricted to zoopathogenic fungi, including the invertebrate pathogens noted in this study, since several species of phytopathogens (i.e. Gaeumannomyces graminis var. tritici, Phytophthora cinnamomi and Cochliobolus sativus) do not survive amoebic phagocytosis, potentially consistent with the lack of macrophage-like cells in host plants that could reinforce such an adaptation (Chakraborty \& Old, 1982; Chakraborty et al., 1983).

Several human pathogenic fungi also share the ability to survive and multiply within amoebae, and similarly it has been hypothesized that this interaction allows these fungi to survive human phagocytic cells (Levitz, 2001). The encapsulated fungus Cryptococcus neoformans is able to survive phagocytosis by amoebae, and replicate within and eventually kill the amoebae, a process similar to its interaction with mammalian macrophages (Levitz, 2001; Bergin et al., 2005). Other human pathogenic fungi, Blastomyces dermatitidis, Sporothrix schenckii and Histoplasma capsulatum, have also been reported to be ingested by amoebae, with phagocytosis resulting in amoeba death and fungal growth (Steenbergen et al., 2004). Interestingly, larvae of the Wax Moth, Galleria mellonella, have been employed as a model system to study C. neoformans pathogenesis, and although the fungus is encapsulated by insect phagocytic haemocytes, infected insects eventually die, suggesting that $C$. neoformans is also able to evade insect haemocyte encapsulation (Mylonakis et al., 2005). Furthermore, some bacterial pathogens that are

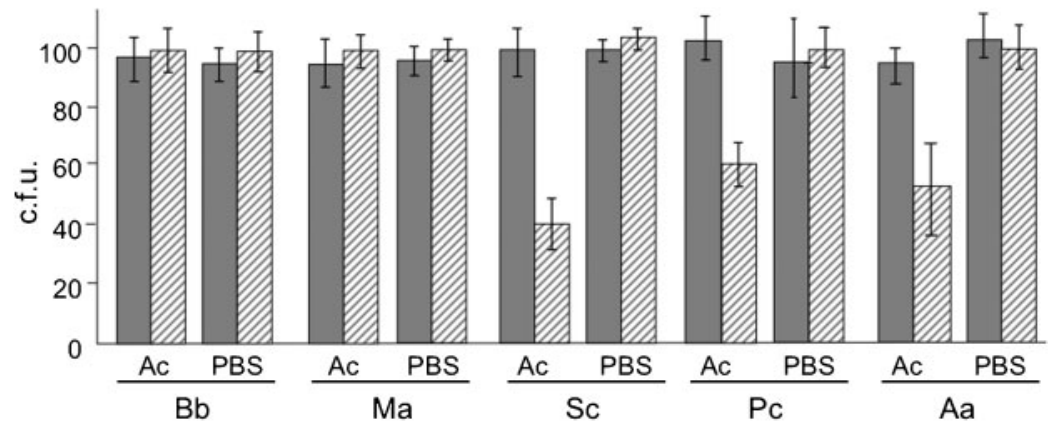

Fig. 7. c.f.u. at $\mathrm{O}$ h (grey bars) and after $72 \mathrm{~h}$ incubation (hatched bars) of $B$. bassiana $(\mathrm{Bb})$, M. anisopliae (Ma), S. cerevisiae (Sc), $P$. chrysogenum (Pc) and Alternaria alternata (Aa) with $A$. castellanii (Ac) or in PBS. Data are representative of three separate experiments, each with $n=5$. Error bars, SD. 


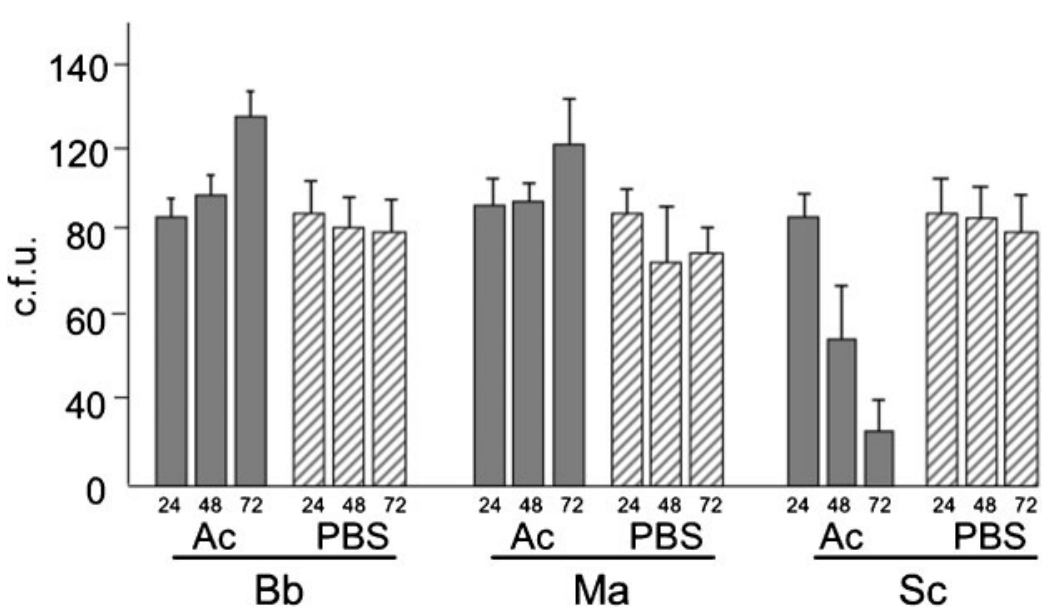

Fig. 8. c.f.u. for three $24 \mathrm{~h}$ cycles (with physical disruption between each cycle) of fungi $[B$. bassiana $(\mathrm{Bb})$, M. anisopliae $(\mathrm{Ma})$ and $S$. cerevisiae (Sc)] incubated with $A$. castellanii (Ac) or in PBS. Data are representative of two separate experiments, each with $n=5$. Error bars, SD. able to survive uptake by macrophages have also been shown to be able to survive amoebic phagocytosis. The interaction with amoebae, both as a replication niche and evolutionary force in adaptation to human pathogenesis, has been noted for Legionella pneumophila, Mycobacterium avium, Listeria monocytogenes, Chlamydia pneumoniae and Burkholderia cepacia (Cirillo et al., 1997; Essig et al., 1997; Ly \& Muller, 1990; Marolda et al., 1999; Swanson \& Hammer, 2000).

Overall, our data support the idea that facultative insect pathogenic fungi such as B. bassiana and M. anisopliae that do not require an insect for survival can maintain certain virulence factors under selective pressures that operate in the soil via predatory amoebae. The interaction with soil amoebae is likely to be only one facet of the multidimensional dynamics of pathogenesis. There are a plethora of additional soil interactions with other fungi, nematodes, protists, bacteria and plants that provide an extremely rich diversity of interactions that we are just beginning to appreciate, and which may contribute to the evolution of virulence factors and the broad insect host ranges of these fungi.

That mammalian as well as insect pathogenic fungi are able to survive amoeba predation is a novel finding. Avoidance of amoeba predation is not just a factor for human pathogens but may be a much more general lifestyle adaptation. The environmental niches, lifestyles and pathogenic consequences of these organisms are radically different, yet they have evolved a similar mechanism for thwarting the amoebae which, consequentially, can be used as a virulence factor for their respective hosts.

\section{ACKNOWLEDGEMENTS}

This research was conducted with the assistance of a Natural Sciences and Engineering Research Council of Canada (NSERC) Discovery Grant to M. J. B. We wish to thank Dr B.-H. Kang (University of Florida, Department of Microbiology and Cell Science) for assistance with the microscopy.

\section{REFERENCES}

Bergin, D., Reeves, E. P., Renwick, J., Wientjes, F. B. \& Kavanagh, K. (2005). Superoxide production in Galleria mellonella hemocytes: identification of proteins homologous to the NADPH oxidase complex of human neutrophils. Infect Immun 73, 4161-4170.

Bidochka, M. J. \& Khachatourians, G. G. (1987). Hemocytic defense response to the entomopathogenic fungus Beauveria bassiana in the migratory grasshopper Melanoplus sanguinipes. Entomol Exp Appl 45, 151-156.

Bidochka, M. J., Kamp, A. M. \& DeCroos, A. M. N. (2000). Insect pathogenic fungi: from genes to populations. In Fungal Pathology, pp. 171-193. Edited by J. W. Kronstad. Dordrecht: Kluwer Academic Press.

Bidochka, M. J., Kamp, A. M., Lavender, T. M., DeKoning, J. \& De Croos, J. N. A. (2001). Habitat association in two genetic groups of the insect-pathogenic fungus Metarhizium anisopliae: uncovering cryptic species? Appl Environ Microbiol 67, 1335-1342.

Bidochka, M. J., Menzies, F. V. \& Kamp, A. M. (2002). Genetic groups of the insect-pathogenic fungus Beauveria bassiana are associated with habitat and thermal growth preferences. Arch Microbiol 178, 531-537.

Chakraborty, S. \& Old, K. M. (1982). Mycophagous soil amoeba: interactions with three plant pathogenic fungi. Soil Biol Biochem 14, 247-255.

Chakraborty, S., Old, K. M. \& Warcup, J. H. (1983). Amoebae from a take-all suppressive soil which feed on Gaeumannomyces graminis tritici and other soil fungi. Soil Biol Biochem 15, 17-24.

Cho, E. M., Boucias, D. \& Keyhani, N. O. (2006a). EST analysis of cDNA libraries from the entomopathogenic fungus Beauveria (Cordyceps) bassiana. II. Fungal cells sporulating on chitin and producing oosporein. Microbiology 152, 2855-2864.

Cho, E. M., Liu, L., Farmerie, W. \& Keyhani, N. O. (2006b). EST analysis of cDNA libraries from the entomopathogenic fungus Beauveria (Cordyceps) bassiana. I. Evidence for stage-specific gene expression in aerial conidia, in vitro blastospores and submerged conidia. Microbiology 152, 2843-2854.

Cirillo, J. D., Falkow, S., Tompkins, L. S. \& Bermudez, L. E. (1997). Interaction of Mycobacterium avium with environmental amoebae enhances virulence. Infect Immun 65, 3759-3767.

Davies, B., Chattings, L. S. \& Edwards, S. W. (1991). Superoxide generation during phagocytosis by Acanthamoeba castellanii: similarities to the respiratory burst of immune phagocytes. J Gen Microbiol 137, 705-710. 
Essig, A., Heinemann, M., Simnacher, U. \& Marre, R. (1997). Infection of Acanthamoeba castellanii by Chlamydia pneumoniae. Appl Environ Microbiol 63, 1396-1399.

Fang, W., Pei, Y. \& Bidochka, M. J. (2006). Transformation of Metarhizium anisopliae mediated by Agrobacterium tumefaciens. Can J Microbiol 52, 623-626.

Gotz, P. \& Boman, H. G. (1985). Insect immunity. In Comprehensive Insect Physiology, Biochemistry and Pharmacology, vol. III, pp. 453485. Edited by G. A. Kerkut \& L. I. Gilbert. Oxford: Pergamon Press.

Holder, D. J. \& Keyhani, N. O. (2005). Adhesion of the entomopathogenic fungus Beauveria (Cordyceps) bassiana to substrata. Appl Environ Microbiol 71, 5260-5266.

Holder, D. J., Kirkland, B. H., Lewis, M. W. \& Keyhani, N. O. (2007). Surface characteristics of the entomopathogenic fungus Beauveria (Cordyceps) bassiana. Microbiology 153, 3448-3457.

Hou, R. F. \& Chang, J. (1985). Cellular defense response to Beauveria bassiana in the silkworm, Bombyx mori. Appl Entomol Zool (Jpn) 20 , $118-125$.

Kurtti, T. J. \& Keyhani, N. O. (2008). Intracellular infection of tick cell lines by the entomopathogenic fungus Metarhizium anisopliae. Microbiology 154, 1700-1709.

Levitz, S. M. (2001). Does amoeboid reasoning explain the evolution and maintenance of virulence factors in Cryptococcus neoformans? Proc Natl Acad Sci U S A 98, 14760-14762.

Ly, T. M. \& Muller, H. E. (1990). Ingested Listeria monocytogenes survive and multiply in protozoa. $J$ Med Microbiol 33, 51-54.

Malliaris, S. D., Steenbergen, J. N. \& Casadevall, A. (2004). Cryptococcus neoformans var. gattii can exploit Acanthamoeba castellanii for growth. Med Mycol 42, 149-158.

Marolda, C. L., Hauroder, B., John, M. A., Michel, R. \& Valvano, M. A. (1999). Intracellular survival and saprophytic growth of isolates from the Burkholderia cepacia complex in free-living amoebae. Microbiology 145, 1509-1517.

Mylonakis, E., Moreno, R., El Khoury, J. B., Idnurm, A., Heitman, J., Calderwood, S. B., Ausubel, F. M. \& Diener, A. (2005). Galleria mellonella as a model system to study Cryptococcus neoformans pathogenesis. Infect Immun 73, 3842-3850.

Old, K. M. \& Darbyshire, J. F. (1978). Soil fungi as food for giant amoebae. Soil Biol Biochem 10, 93-100.

Pendland, J. C., Hung, S. Y. \& Boucias, D. G. (1993). Evasion of host defense by in vivo-produced protoplast-like cells of the insect mycopathogen Beauveria bassiana. J Bacteriol 175, 5962-5969.

Roberts, D. W. \& Hajek, A. E. (1992). Entomopathogenic fungi as bioinsecticides. In Frontiers of Industrial Mycology, pp. 144-159. Edited by G. F. Leatham. New York: Chapman and Hall.

Scully, L. R. \& Bidochka, M. J. (2006). Developing insect models for the study of current and emerging human pathogens. FEMS Microbiol Lett 263, 1-9.

Steenbergen, J. N., Shuman, H. A. \& Casadevall, A. (2001). Cryptococcus neoformans interactions with amoebae suggest an explanation for its virulence and intracellular pathogenic strategy in macrophages. Proc Natl Acad Sci U S A 98, 15245-15250.

Steenbergen, J. N., Nosanchuk, J. D., Malliaris, S. D. \& Casadevall, A. (2004). Interaction of Blastomyces dermatitidis, Sporothrix schenckii, and Histoplasma capsulatum with Acanthamoeba castellanii. Infect Immun 72, 3478-3488.

Swanson, M. S. \& Hammer, B. K. (2000). Legionella pneumophila pathogenesis: a fateful journey from amoebae to macrophages. Annu Rev Microbiol 54, 567-613.

Wang, C. \& St. Leger, R. J. (2006). A collagenous protective coat enables Metarhizium anisopliae to evade insect immune responses. Proc Natl Acad Sci U S A 103, 6647-6652.

Wang, C., Hu, G. \& St. Leger, R. J. (2005). Differential gene expression by Metarhizium anisopliae growing in root exudate and host (Manduca sexta) cuticle or hemolymph reveals mechanisms of physiological adaptation. Fungal Genet Biol 42, 704-718.

Zimmermann, G. (1986). The "Galleria bait method" for detection of entomopathogenic fungi in soil. J Appl Entomol 102, 212-215.

Edited by: B. A. Horwitz 